\title{
Infrared spectroscopic study of triacetyl- $\beta$-cyclodextrin and its inclusion complex with nicardipine
}

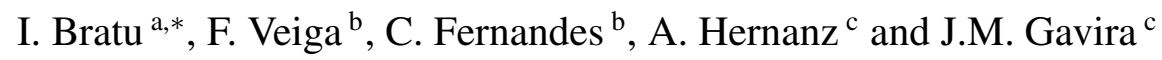 \\ ${ }^{a}$ National Institute for Research and Development of Isotopic and Molecular Technologies, \\ P.O. Box 700, R-3400 Cluj-Napoca 5, Romania \\ ${ }^{\mathrm{b}}$ Laboratory of Pharmaceutical Technology, Faculty of Pharmacy, University of Coimbra, Coimbra, \\ Portugal \\ ${ }^{\mathrm{c}}$ Universidad Nacional de Educación a Distancia, Departamento de Ciencias y Tecnicas Físico \\ Quimicas, Senda del Rey 9, 28040 Madrid, Spain
}

\begin{abstract}
Infrared spectra of inclusion compounds of triacetyl- $\beta$-cyclodextrin with nicardipine hydrochloride were compared and analysed with those corresponding to their physical mixture and the pure compounds, respectively. Different O-H stretching vibrations, assigned to water molecules, were located in the Fourier Transform Infrared (FT IR) spectra of triacetyl- $\beta$ cyclodextrin, and its inclusion complex with nicardipine obtained by spray-drying (SD) method. Water molecules involved in various hydrogen bonds environments change their status during complexation process. Evidences are observed of the formation of the complex especially in the spectral regions of the amino and carbonyl stretching vibrations.
\end{abstract}

Keywords: FT IR spectra, self-deconvolution, inclusion complex, nicardipine, triacetyl- $\beta$-cyclodextrin

\section{Introduction}

Cyclodextrins (CDs) act as molecular hosts for a large variety of guest molecules, polar and non-polar ones. In order to increase their host qualities, derivatives of CDs were prepared, among them triacetyl$\beta$-cyclodextrin (TA- $\beta$-CD). This molecule has a rigid cavity similar to the one of $\beta$-CD, but all the $\mathrm{OH}$ groups of $\beta$-CD are replaced by $\mathrm{OCOCH}_{3}$ groups. It is interesting to make a comparative spectroscopic study concerning these two CDs. In the case of pure $\beta$-CD $[1,2]$ both infrared and Raman spectra in the 4000-3000 $\mathrm{cm}^{-1}$ region were reported and analysed, whereas for $\beta$-CD complexes only the FT IR spectra of copper(II)- $\beta$-CD has been investigated [3] in this spectral region. FT IR, X-ray diffraction and thermal studies [4-6] were used in the structure investigation of different inclusion complexes in solid state. Some interesting studies were reported on the hydration and dehydration processes [2,7] and thermal stability of $\beta$-CD [8] or hydrophobically modified $\beta$-CD [9]. In order to clarify the nature of the bands that appear in the $4000-3000 \mathrm{~cm}^{-1}$ spectral region, infrared spectra at low temperature (liquid nitrogen and liquid helium) were reported [10]. From these experimental data, Fourier self-deconvolution and band fitting procedures, the number of the components in the $4000-3000 \mathrm{~cm}^{-1}$ spectral region was determined and an assignment of these $\mathrm{O}-\mathrm{H}$ stretching bands appearing there was performed [1]. Until now, there are no publication, to our knowledge, on TA- $\beta$-CD and its inclusion complexes by FT IR

\footnotetext{
${ }^{*}$ Corresponding author. E-mail: ibratu@s3.itim-cj.ro.
} 


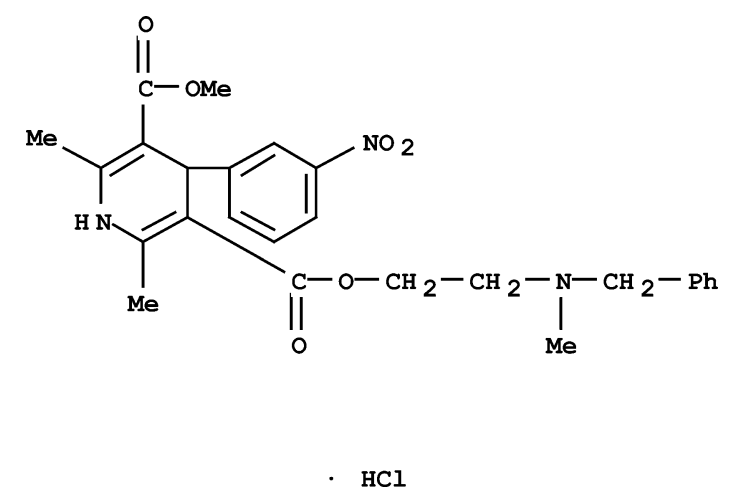

Fig. 1. Structural formula of nicardipine hydrochloride.

spectroscopy. This paper presents the results of an analysis applied to this case where its inclusion complex contains nicardipine hydrochloride (NIC), Fig. 1, a calcium channel-blocking agent used in the management of mild to moderate hypertension, angina pectoris and cerebral disease.

\section{Experimental}

Nicardipine hydrochloride from Effechem SRL (Milan, Italy) and TA- $\beta$-CD from Aldrich were used without further purification. Equimolar quantities of NIC and TA- $\beta$-CD were dissolved in ethanol : water $(50: 50)$. The resulting mixture was stirred for $24 \mathrm{~h}$ at room temperature and the obtained solution was subsequently spray-dried (LabPlant SD-05), under the following conditions: $50 \mathrm{~m}^{3} / \mathrm{h}$ - air flow, $1.0 \mathrm{bar}$ - atomising air pressure, $160^{\circ} \mathrm{C}$ - inlet temperature, $85^{\circ} \mathrm{C}$ - outlet temperature and $400 \mathrm{ml} / \mathrm{h}$ - flow rate of solution. The product was sieved through a $63-160 \mu \mathrm{m}$ mesh. A physical mixture (PM) of NIC and TA- $\beta$-CD (1:1 molar ratio) was also prepared.

The FT IR spectra were obtained using the $\mathrm{KBr}$ pellet technique, $0.8 \mathrm{mg}$ of sample in $300 \mathrm{mg}$ of $\mathrm{KBr}$. They were collected with a Bomem DA3 FT IR spectrometer, working under a vacuum (pressure $\leqslant 133.3 \mathrm{~Pa}$ ) to avoid IR absorption from atmospheric water. The effective apodized resolution was $s=1.77 \mathrm{~cm}^{-1}$ (RES $=2$ and Hamming apodizing function). Good S/N ratios were obtained by coadding 250 interferograms. Different procedures for obtaining the Fourier self-deconvolution of the FT IR spectra have been proposed recently $[11,12]$. Our work was done with the so-called reference Fourier self-deconvolution procedure (RFSD) [12]. Second derivatives of the FT IR spectra and curve fitting were obtained with Thermo Galactic GRAMS/32 software.

\section{Results and discussion}

\section{1. $4000-2000 \mathrm{~cm}^{-1}$}

This spectral region, where the $\mathrm{OH}$ stretching vibrations appear, presents an overlapping of primary and secondary $\mathrm{OH}$ group vibrations in $\beta-\mathrm{CD}$, bonded intra- or inter-molecularly. Applying deconvolution techniques to the FT IR spectra of $\beta-\mathrm{CD}$ and based on the results obtained previously $[1,3]$, it has been observed in this region that a superposition of the $\mathrm{OH}$ stretching vibrations corresponding to interstitial and intra-cavity water molecules occurs together with those assigned to the primary and secondary 

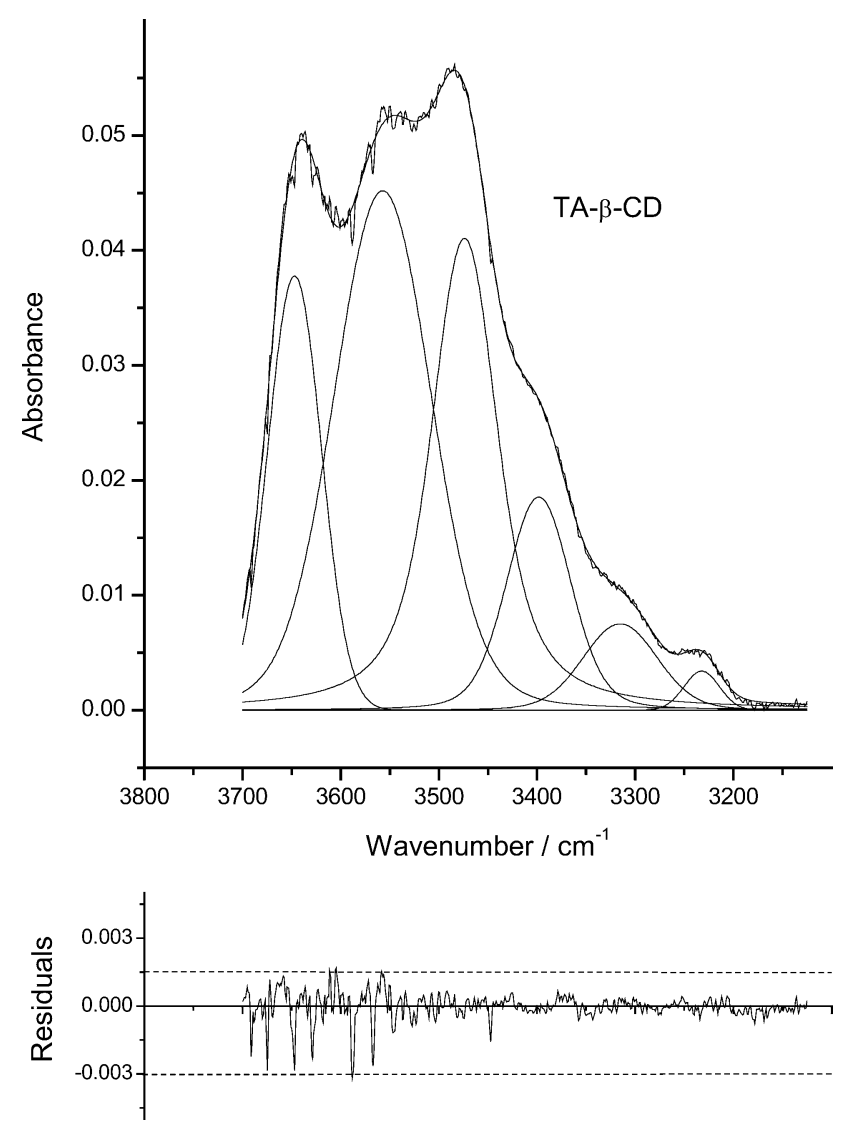

Fig. 2. $\mathrm{O}-\mathrm{H}$ stretching region for $\mathrm{TA}-\beta-\mathrm{CD}$.

$\mathrm{OH}$ groups of the $\beta$-CD. In the case of TA- $\beta$-CD there are no primary and secondary $\mathrm{OH}$ groups, being replaced by $\mathrm{OCOCH}_{3}$ groups, therefore the spectrum must reflect this change. Indeed, the FT IR spectra of TA- $\beta$-CD, see Fig. 2, of the inclusion complex (Fig. 3) and of the physical mixture (Fig. 4), do not contain the bands that correspond to primary and secondary $\mathrm{OH}$ groups [1,3]. It should be remarked the low absorbance values in this spectral region in the case of TA- $\beta$-CD, whereas for $\beta$-CD $[1,3]$ the corresponding absorbance values are ten times higher for the same concentration in $\mathrm{KBr}$ pellet.

Nevertheless, some very weak bands appear above $3400 \mathrm{~cm}^{-1}$, Fig. 2. They should be assigned to $\mathrm{O}-\mathrm{H}$ stretching vibrations of water molecules involved in different hydrogen bonding schemes [1,3]. By applying Fourier self-deconvolution techniques [12] and second derivative computations the wavenumber of the maxima for the band components have been evaluated. Curve fitting procedures with Voigt functions were applied to the experimental profiles based on these wavenumber values. Relative integrated intensities of the band components, $I_{\text {rel }}$, were calculated as the ratios of each integrated intensity $v s$. the sum of the component intensities, Table 1 and Figs 2-5. Relevant changes of intensity were observed for the components at $\sim 3480$ and $\sim 3420 \mathrm{~cm}^{-1}$ for PM and SD, Table 1 and Figs 3 and 4 . The significant decrease of intensity for the component at $\sim 3480 \mathrm{~cm}^{-1}$ and the increase for the component at $\sim 3420 \mathrm{~cm}^{-1}$ on complexation suggest a change of the number of water molecules involved in the hydrogen bond scheme. A redistribution of the water molecules among the different hydrogen bonds sites could take place. As dehydration of $\beta$-CD affects the water molecules included in its cavity to a 


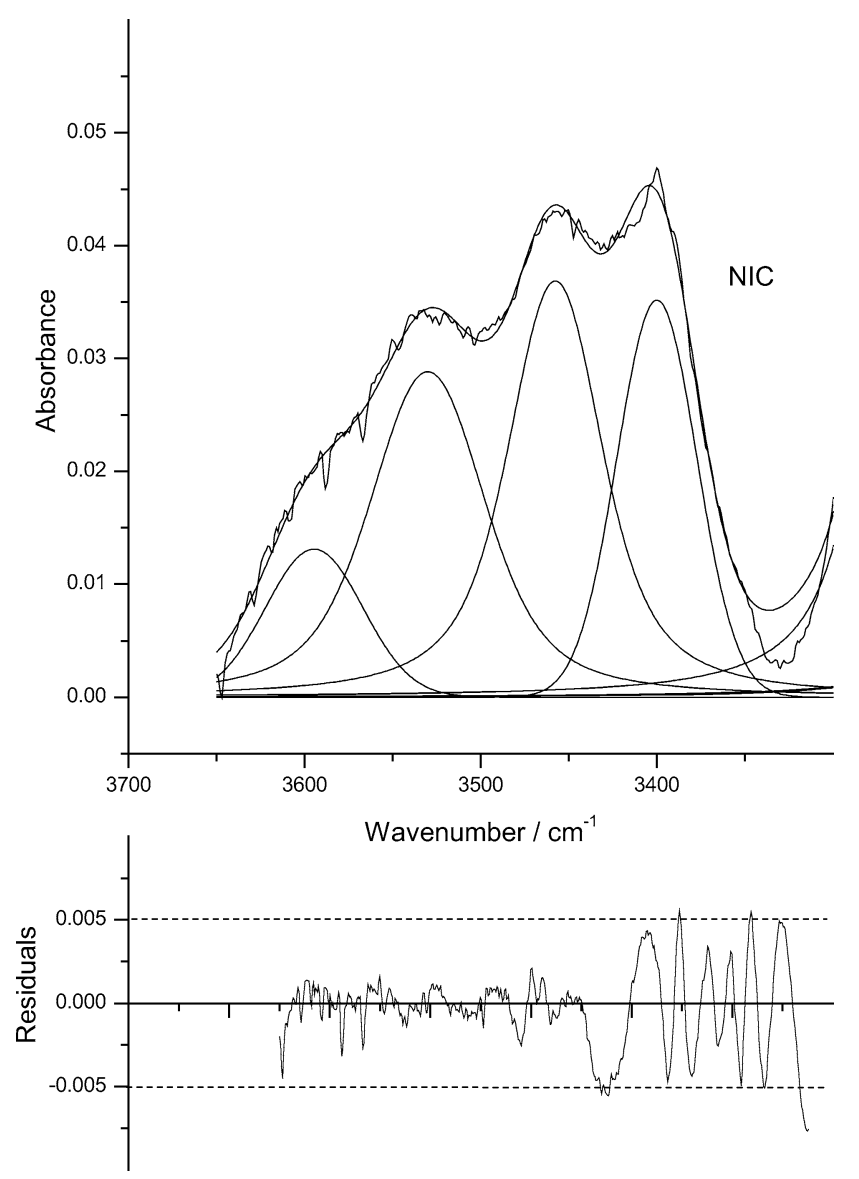

Fig. 3. O-H stretching region for NIC.

larger extent than the interstitial ones [13], it would be inferred that during the complexation process the drug enters into the TA- $\beta$-CD torus-shaped cavity and the water molecules from the inside of the torus change their hydrogen bonding environment.

An assignment of the bands that are changed upon complexation process is done and a possible explanation for these spectral changes is given. As concerning the functional groups of the NIC, one can observe, see Fig. 6, a medium intensity band at $3253 \mathrm{~cm}^{-1}$, probably assigned to $\mathrm{N}-\mathrm{H}$ stretching vibration [14-16] and a strong doublet band at 3182 and $3199 \mathrm{~cm}^{-1}$, assigned to $\mathrm{C}-\mathrm{H}$ stretching vibration. These bands are also observed in the spectrum of the physical mixture but in the case of the spray drying compound (SD), their intensities diminish considerably, specially the one located at $3253 \mathrm{~cm}^{-1}$, the other two weakens and broadens clearly. On going to lower frequencies, the structured bands of NIC in the $2700-2300 \mathrm{~cm}^{-1}$ range are probably due to $(\mathrm{NH}-\mathrm{N})$ stretching bands. This structure of bands is also observed in the spectrum of PM but it is quite difficult to be observed in the spectrum of SD, a possible explanation being the breaking of these intermolecular links.

\section{2. $2000-700 \mathrm{~cm}^{-1}$}

Comparing the spectra of NIC and TA- $\beta$-CD in this spectral region, see Fig. 7, a strong band of NIC may be observed at $1707 \mathrm{~cm}^{-1}$ with a shoulder at $1704 \mathrm{~cm}^{-1}$, both due to the $\nu_{\mathrm{C}=\mathrm{O}}$ vibration of the ester 


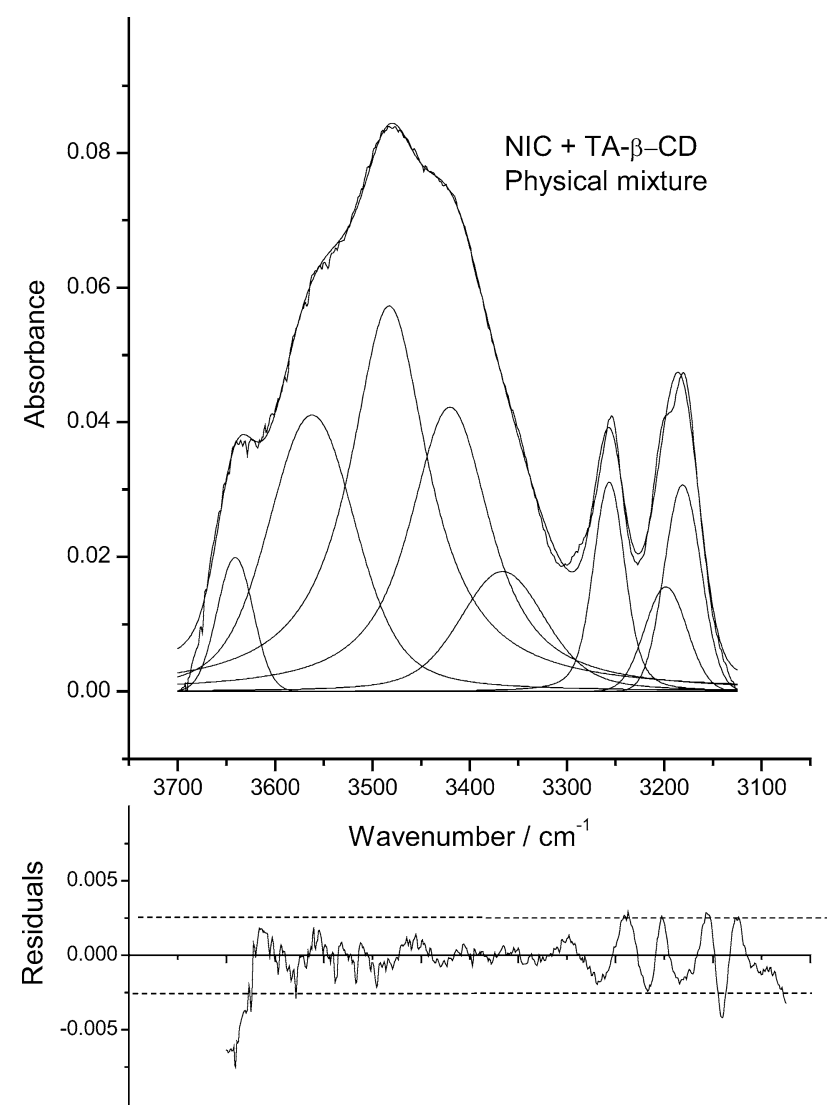

Fig. 4. FT IR spectrum of the physical mixture NIC + TA- $\beta-\mathrm{CD}, 1: 1$ molar.

groups. However, a strong band is observed in the TA- $\beta$-CD spectrum at $1757 \mathrm{~cm}^{-1}$, but in this case due to $\nu_{\mathrm{C}=\mathrm{O}}$ vibration of the acetyl group.

In the spectrum of PM both bands are observed, whereas in the SD spectrum the $\nu_{\mathrm{C}=\mathrm{O}}$ band of the ester groups of NIC is shifted to lower frequencies $\left(1701 \mathrm{~cm}^{-1}\right)$, diminished in intensity and broadened. Similar effects were observed and explained previously [17] for the HP- $\beta$-CD complex of NIC (NICHP- $\beta$-CD), suggesting the formation of hydrogen bonds between the carbonyl groups of NIC and the hydroxyl groups of the host cavity during inclusion phenomena. These observations are in full agreement with other authors [18] who reported that when a carbonyl group is joined to a hydroxyl by hydrogen bonds, the stretching band is shifted to lower frequencies due to the weakening of the carbonyl radical double bond. In this case, hydrogen bonds may be formed with water molecules present in the cavity. That is, a weakening of the double bond in the carbonyl group appears.

Phenyl group vibration (ring carbon-carbon stretching) appears at $1623 \mathrm{~cm}^{-1}$ in the spectrum of NIC, but it diminishes considerably of intensity in the spectrum of SD.

About the $\mathrm{NO}_{2}$ group vibrations, see Fig. 8, their bands are located at $1536 \mathrm{~cm}^{-1}, \nu_{\mathrm{as}}\left(\mathrm{NO}_{2}\right)$, and $1354 \mathrm{~cm}^{-1}, \nu_{\mathrm{s}}\left(\mathrm{NO}_{2}\right)$, respectively. The band at $1494 \mathrm{~cm}^{-1}$ is probably due to the phenyl stretching vibration. Upon complexation the $\nu_{\text {as }}\left(\mathrm{NO}_{2}\right)$ band is shifted to lower frequencies, $1530 \mathrm{~cm}^{-1}$, and the $\nu_{\mathrm{s}}\left(\mathrm{NO}_{2}\right)$ is shifted to $1350 \mathrm{~cm}^{-1}$, whereas the phenyl band of SD $\left(\sim 1490 \mathrm{~cm}^{-1}\right)$ is less affected by this process. 
Table 1

Spectral treatment of the $3800-3100 \mathrm{~cm}^{-1}$ region: wavenumber of the band maxima obtained by spectral deconvolution, their corresponding relative integrated intensities $\left(I_{\text {rel }}\right)$ and absorbances $\left(A_{\max }\right)$ evaluated by curve fitting

\begin{tabular}{|c|c|c|c|c|c|c|c|}
\hline \multicolumn{8}{|c|}{ NIC + TA- $\beta$-CD, physical mixture } \\
\hline$\nu / \mathrm{cm}^{-1}$ & 3640 & 3562 & 3482 & 3420 & 3366 & & \\
\hline$A_{\max }$ & 0.020 & 0.041 & 0.057 & 0.042 & 0.017 & & \\
\hline$I_{\text {rel }}$ & 0.043 & 0.233 & 0.360 & 0.262 & 0.102 & & \\
\hline \multicolumn{8}{|c|}{$\mathrm{NIC}+\mathrm{TA}-\beta-\mathrm{CD}$, spray drying } \\
\hline$\nu / \mathrm{cm}^{-1}$ & 3638 & 3559 & 3477 & 3419 & 3368 & 3266 & 3201 \\
\hline$A_{\max }$ & 0.027 & 0.058 & 0.048 & 0.047 & 0.026 & 0.019 & 0.013 \\
\hline$I_{\text {rel }}$ & 0.064 & 0.290 & 0.224 & 0.305 & 0.116 & & \\
\hline \multicolumn{8}{|c|}{ TA- $\beta-\mathrm{CD}$} \\
\hline$\nu / \mathrm{cm}^{-1}$ & 3646 & 3557 & 3473 & 3398 & 3315 & 3232 & \\
\hline$A_{\max }$ & 0.0377 & 0.0451 & 0.041 & 0.0185 & 0.007 & 0.0034 & \\
\hline$I_{\text {rel }}$ & 0.169 & 0.390 & 0.283 & 0.109 & 0.050 & & \\
\hline \multicolumn{8}{|c|}{ NIC } \\
\hline$\nu / \mathrm{cm}^{-1}$ & 3594 & 3530 & 3457 & 3399 & & & \\
\hline$A_{\max }$ & 0.0131 & 0.0288 & 0.0368 & 0.0351 & & & \\
\hline$I_{\mathrm{rel}}$ & 0.103 & 0.314 & 0.353 & 0.230 & & & \\
\hline
\end{tabular}
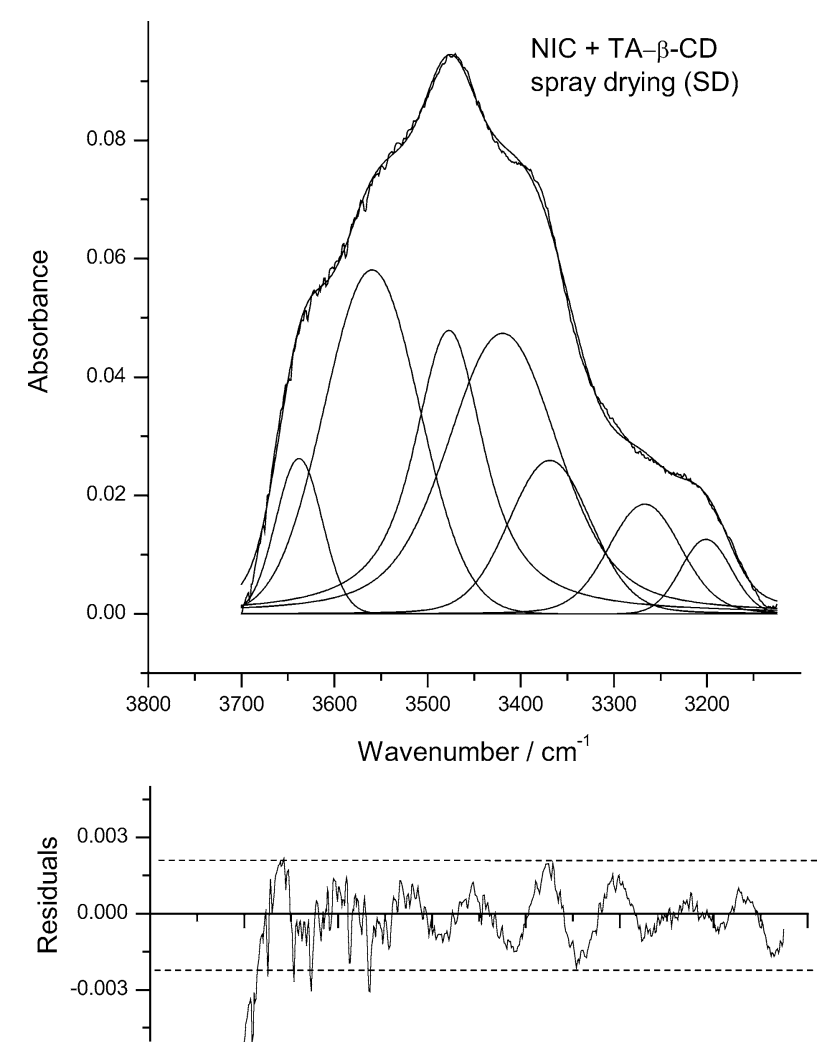

Fig. 5. O-H stretching region for the spray drying product. 


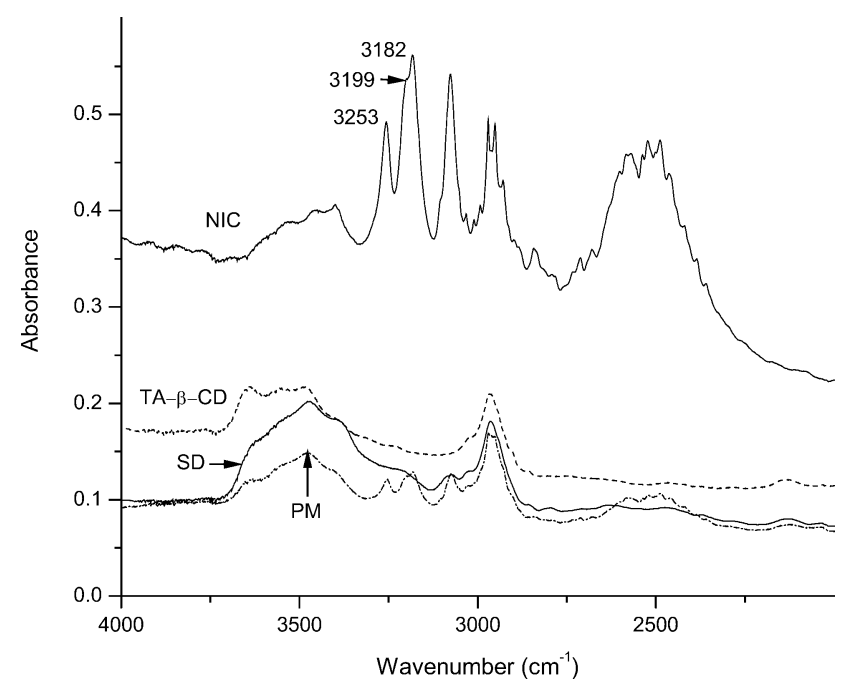

Fig. 6. FT IR spectra $\left(4000-2000 \mathrm{~cm}^{-1}\right)$ of NIC, TA- $\beta$-CD, PM and SD.

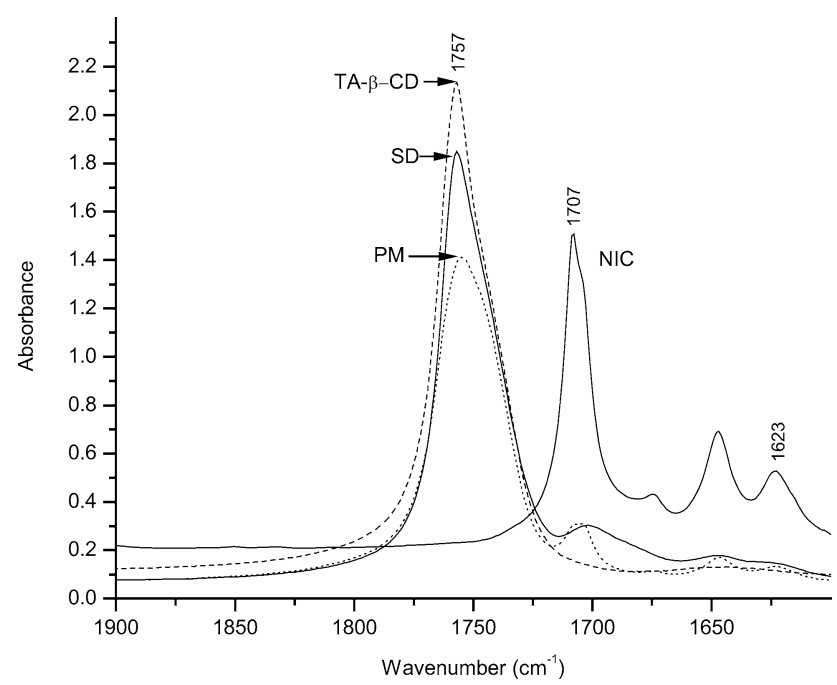

Fig. 7. FT IR spectra $\left(2000-1600 \mathrm{~cm}^{-1}\right)$ of NIC, TA- $\beta-C D, P M$ and SD.

The band at $789 \mathrm{~cm}^{-1}$, see Fig. 9, in the spectrum of NIC and assigned to vibrations of the $-\mathrm{CH}_{2}-$ group is shifted to lower frequencies $\left(782 \mathrm{~cm}^{-1}\right.$ ) and broadens in SD spectrum. The band at $745 \mathrm{~cm}^{-1}$ is unchanged in position upon complexation but broadens.

Finally, the doublet band at 711 and $700 \mathrm{~cm}^{-1}$ in NIC and PM spectra, become a broad band centred at $704 \mathrm{~cm}^{-1}$ in SD spectrum.

From this spectral analysis it can be concluded that the most sensitive functional groups, involved in the complexation process, are the carbonyl and the nitro groups. The NH group is also affected by possible host/guest hydrogen bond formation. The formation of the CD inclusion complexes is usually accompanied [19] with the lost of intracavity water molecules. The release of water molecules from the CD cavity into the gas phase is considered a thermodynamic step in CD complexation [20]. 


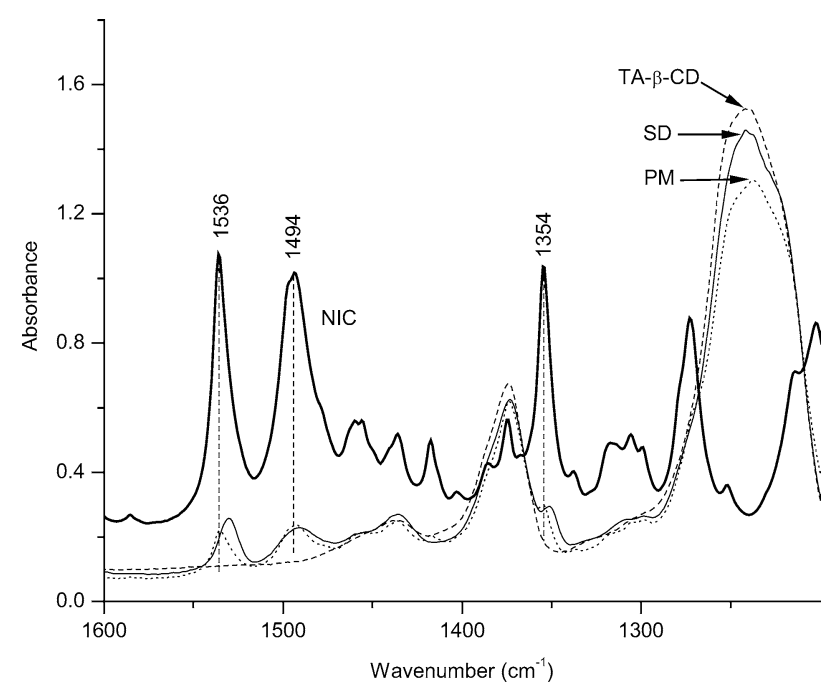

Fig. 8. FT IR spectra $\left(1600-1200 \mathrm{~cm}^{-1}\right)$ of NIC, TA- $\beta$-CD, PM and SD.

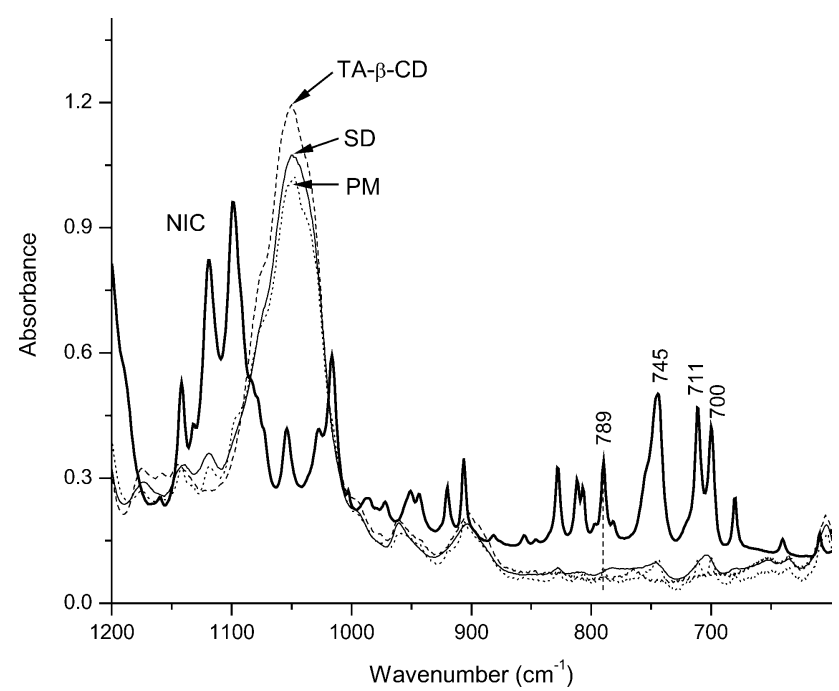

Fig. 9. FT IR spectra $\left(1600-600 \mathrm{~cm}^{-1}\right)$ of NIC, TA- $\beta-C D$, PM and SD.

\section{Acknowledgements}

One of us (I.B.) is grateful to the NATO Science Committee for a D.c type grant supporting his scientific stay in UNED, Madrid. Thanks are due to Dr. N.T. Kotchev for offering the software "Program for Spectra Deconvolution" programmed by N.T. Kotchev (Plovdiv), M.I. Rogojerov (Sofia) and G.N Andreev (Plovdiv), Bulgaria. The authors are also grateful to a reviewer for comments that improved this work. 


\section{References}

[1] O. Egyed, Vibr. Spectrosc. 1 (1990), 225.

[2] J.M. Gavira, A. Hernanz and I. Bratu, Vibr. Spectrosc. 32 (2003), 137.

[3] O. Egyed and V. Weiszfeiler, Vibr. Spectrosc. 7 (1994), 73.

[4] M.K. Rotich, M.E. Brown and B.D. Glass, Journal of Thermal Anal. and Calorim. 73(2) (2003), 687.

[5] M.K. Rotich, M.E. Brown and B.D. Glass, Journal of Thermal Anal. and Calorim. 73(2) (2003), 671.

[6] M.R. Caira, E.J.C. de Vries and L.R. Nassimbeni, Journal of Thermal Anal. and Calorim. 73(2) (2003), 647.

[7] A.M.G. Moreira Da Silva, Th. Steiner, W. Saenger, J.M.A. Empis and J.J.C. Teixeira-Dias, J. of Incl. Phenom. and Molec. Recogn. in Chem. 25 (1996), 21.

[8] Y.Q. Xu, J.M. Tang, Q. Zhou, Z. Qin and S.Q. Sun, Chinese J. Anal. Chem. 31 (5) (2003), 590.

[9] F. Witte and H. Hoffmann, J. of Incl. Phenom. and Molec. Recogn. in Chem. 25 (1996), 25.

[10] E.V. Korolik, N.V. Ivanov, V.V. Sivchik, R.G. Jbankov and N.I. Istratova, Journ. Prikl. Spektrosk. 34 (1981), 855.

[11] V.A. Lorenz-Fonfria, J. Villaverde and E. Padros, Appl. Spectrosc. 56 (2002), 232.

[12] N.T. Kochev, M.I. Rogojerov and G.N. Andreev, Vibr. Spectrosc. 25 (2001), 177.

[13] T. Steiner and G. Koellner, J. Amer. Chem. Soc. 116 (1994), 5122.

[14] N.B. Colthup, L.H. Daly and S.E. Wiberley, Introduction to Infrared and Raman Spectroscopy, Acad. Press, New York, 1975.

[15] N.P.G. Roeges, A Guide to Complete Interpretation of Infrared Spectra of Organic Structures, Wiley \& Sons, Chichester, 1994.

[16] G. Socrates, Infrared Characteristic Group Frequencies, Wiley \& Sons, Chichester, 1980.

[17] C.M. Fernandes, M.T. Veira and F.J.B. Veiga, Eur. J. Pharm. Sci. 15 (2002), 79.

[18] F. Otero-Espinar, S. Anguiano-Igea, S. Garcia-Gonzales, J. Vila-Jato and J. Blanco-Mendez, Int. J. Pharm. 79 (1992), $149-157$.

[19] K.H. Frömming and J. Szejtli, Cyclodextrins in Pharmacy (Topics in Inclusion Science, Vol. 5), Kluwer Academic Publishers, Dordrecht, The Netherlands, 1994, pp. 50-51.

[20] L. Liu and Q.-X. Guo, J. of Inclusion Phenomena and Macrocyclic Chemistry 42 (2002), 1. 


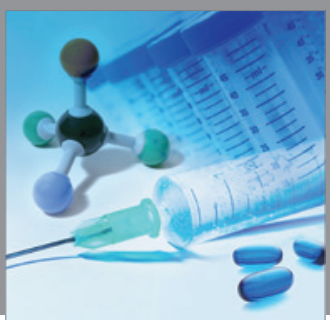

International Journal of

Medicinal Chemistry

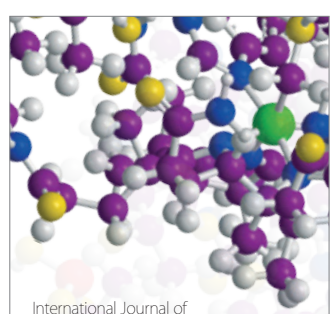

Carbohydrate Chemistry

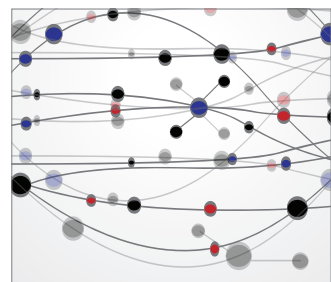

The Scientific World Journal
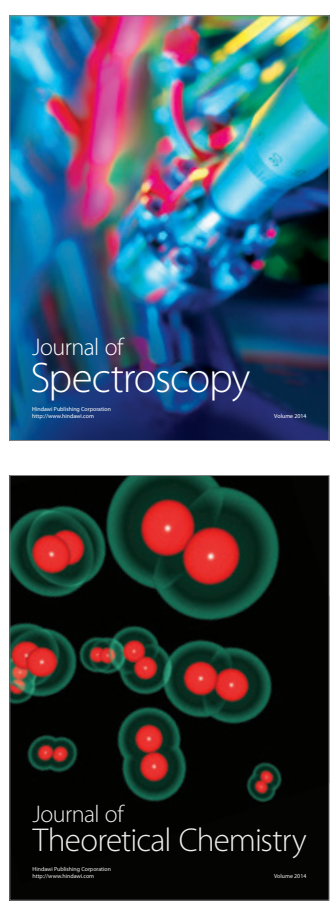
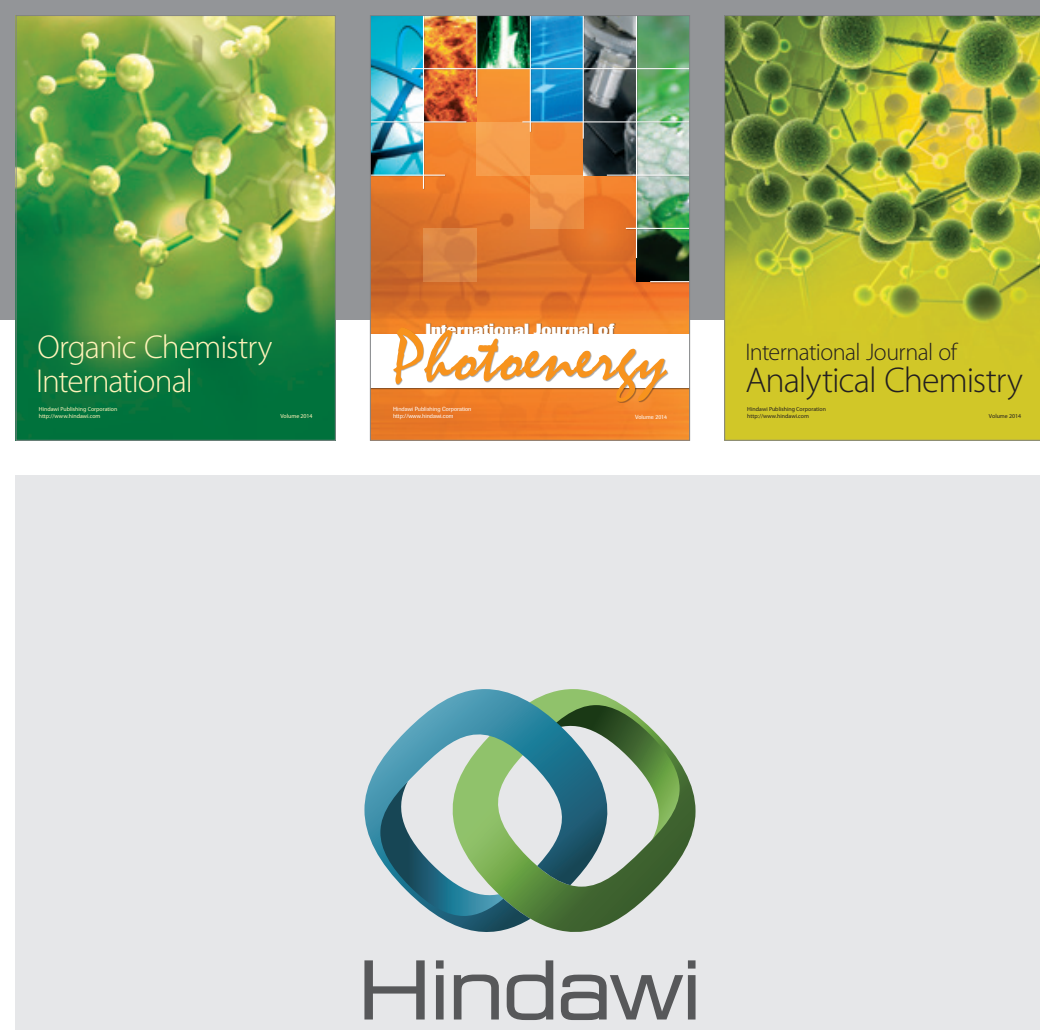

Submit your manuscripts at

http://www.hindawi.com
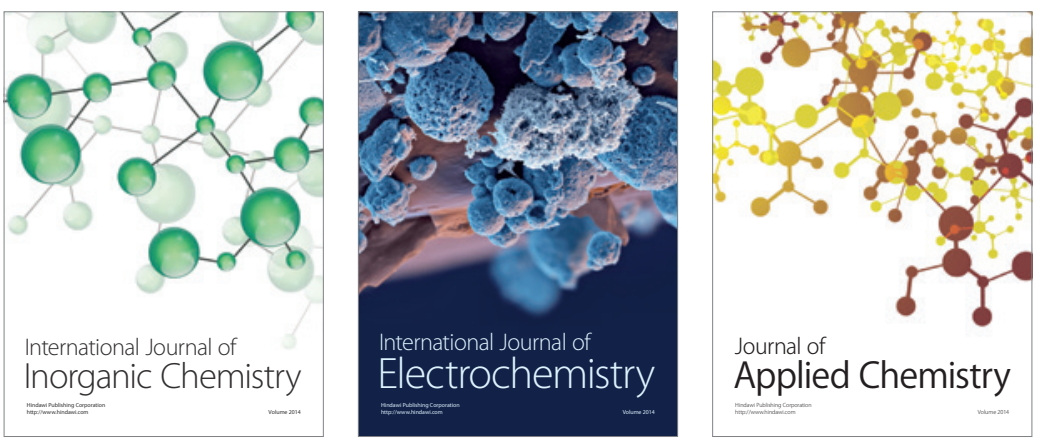

Journal of

Applied Chemistry
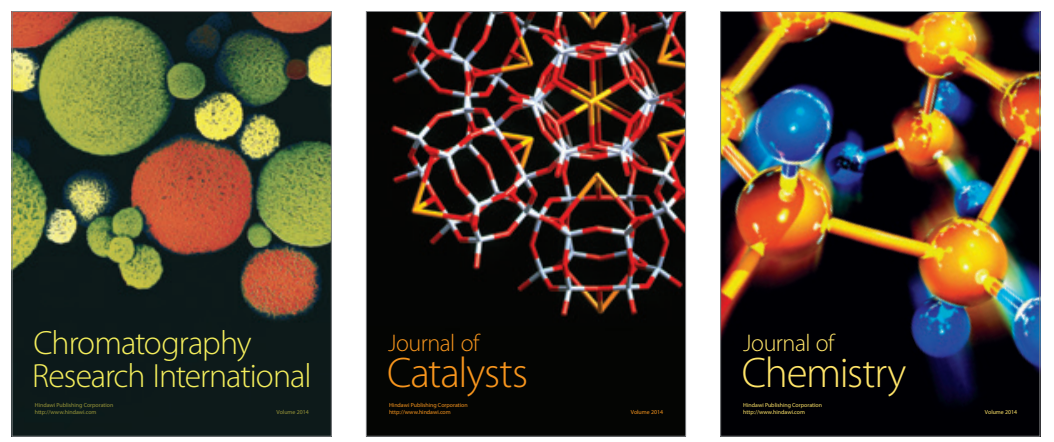
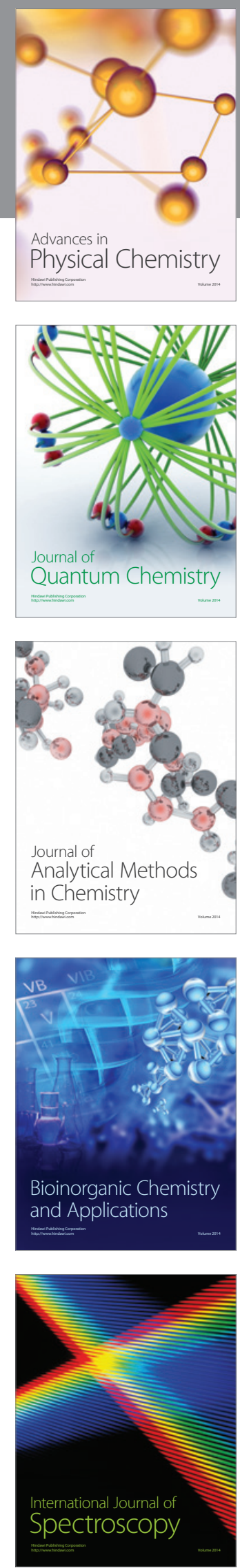